\title{
The Development of a Questionnaire to Assess the Attitude of Active Listening
}

\author{
Norio Mishima', Shinya Kubota ${ }^{2}$ and Shoji Nagata ${ }^{1}$ \\ 'Department of Mental Health, Institute of Industrial Ecological Sciences and \\ ${ }^{2}$ Occupational Health Training Center, University of Occupational and Environmental Health (UOEH)
}

\begin{abstract}
The Development of a Questionnaire to Assess the Attitude of Active Listening: Norio Mishima, et al. Department of Mental Health, Institute of Industrial Ecological Sciences-Interpersonal relationships are widely recognized as a major source of job stress, and a mental health training program that incorporates active listening $(\mathrm{AL})$ has attracted much attention in Japan. However, few methods are available to assess the attitude of AL easily. To develop a new questionnaire that can measure workers' personcentered attitude (PCA), which is closely related to $A L$, we formulated a scale of 47 items that describe basic elements of the PCA and AL. This preliminary scale was administered to 536 workers (426 male and 95 female) at two manufacturing companies. Responses to the scale items were subjected to a principal factor analysis followed by a varimax rotation. A scree test suggested three meaningful factors, whose cumulative contribution was $81.2 \%$. These factors were subsequently labeled as "Listening Attitude", "Listening Skill", and "Conversation Opportunity". Both Cronbach's alphas and test-retest reliabilities indicated that all three factors were highly reliable. To examine the validity of the scale, factor scores of lay persons (middle-aged workers) were compared with those of 39 clinical therapists who specialize in psychosomatic medicine. Scores for all three factors were significantly higher for the therapists than for the lay persons. Of the three factors derived from the preliminary scale, the first and second showed remarkable differences between the groups and were retained for their usefulness for evaluating AL. The new scale, the Active Listening Attitude Scale (ALAS), is expected to be a useful instrument for various mental health programs to assess the PCA.
\end{abstract}

(J Occup Health 2000; 42: 111-118)

Received Aug 23, 1999; Accepted Dec 13, 1999

Correspondence to: N. Mishima, Department of Mental Health Institute of Industrial Ecological Sciences University of Occupational and Environmental Health (UOEH), I-1 Iseigarka, Yahatanishi-ku, Kitakyushu 807-8555, Japan
Key words: Active listening, Person-centered attitude, Questionnaire, Factor analysis, Reliability, Validity

The number of workers who are under stress in the workplace has been increasing. In Japan, nationwide surveys on employee health conducted in 1982, 1987, and 1992 by the Ministry of Labour indicated that the percentage of employees experiencing work-related stress gradually increased from $50.6 \%$ in 1982 to $57.3 \%$ in $1992^{1 "}$. In particular, the problem of "human relationships in the workplace" was associated with increasing stress levels among several subgroups of employees, such as 50-59-year-old males and 40-49-year-old females. When taking into account the influence of stress on workers" health, these results show that stress management in the workplace is very important to maintain workers' health.

A NIOSH job-stress model identified various psychosocial conditions in the workplace, such as interpersonal conflict and role ambiguity, as stressors at work ${ }^{21}$. Based on this model, improving interpersonal relationships in the workplace is expected to reduce the level of job stress. Ikemi et al. studied the relationship between the mental health of 1,661 workers and the person-centered attitude (PCA) levels of their superiors by using a questionnaire ${ }^{3)}$. They found that workers who reported that their superiors had a high PCA had significantly less fatigue, depression and anxiety than those who perceived of their superiors as having a low PCA. They concluded that the PCA of job superiors was considered to be positively related to the mental health of workers.

The American psychologist Carl R. Rogers first defined the $\mathrm{PCA}^{4)}$. It is considered to be the basic interpersonal attitude that is required of counselors and clinical psychologists ${ }^{5}$. He also advocated that, when constructive human relationships are observed among a group of ordinary people, the PCA plays a significant role in creating those relationships. It consists of three main elements: "empathic understanding", "unconditional 
positive regard", and "congruence". Listening to a person in this attitude is called "active listening" (AL) $)^{6}$.

Since the beginning of the 1990 s, AL has been considered to be a useful method for reducing stress in relationships among workers. The Total Health Promotion Plan (THP) of the Japan Ministry of Labour introduced it as an important component of its psychological consultation ${ }^{7}$. However, in spite of the popularity of AL, as far as we know, the study conducted by Kubota et al. ${ }^{81}$ is the only one that discusses how to measure the direct effects of AL training among workers.

Kubota et al. reported on their corporate mental health training program, which incorporated AL in its core training, and compared participants' attitudes before and after the training by using a questionnaire they had developed. They found significant changes in some items, and concluded that their program helped participants to acquire the basic attitudes of the PCA. Although the internal reliability of the questionnaire based on Cronbach's alpha was high, it had not been standardized before their study and its validity was not reported. The questionnaire has therefore not yet been verified as an appropriate instrument for regular use in the workplace.

In the United States, Gendlin ${ }^{9)}$ and Klein et al. ${ }^{(0)}$ developed the 'experiencing' scale to assess the process and depth of experience that people would encounter during AL. Ikemi et al. developed the Japanese version of this scale ${ }^{11}$. However, this scale is a rating given by an observer who carefully watches the conversation process rather than a self-administered questionnaire. The process was evaluated by selecting one of seven categories in the scale that best described the level of experiencing observed. Because much training and experience are required to rate conversations in this way, it is not appropriate for lay workers to use the experiencing scale to evaluate their own level of AL in the workplace.

To the best of our knowledge, there are no convenient instruments for measuring the PCA easily. Although Japanese researchers and workplace healthcare professionals have paid much attention to AL, few studies have assessed its usefulness for managing stress objectively. The lack of an instrument may be one of the major reasons for this. We therefore believe that developing a questionnaire to evaluate listeners' attitudes related to the PCA and AL is very important in order to promote a mental health program using AL. Our new questionnaire, the Active Listening Attitude Scale (ALAS), will be a useful tool for evaluating the effectiveness of training programs. In this study we report the results of factor analysis of the ALAS, and the reliability and validity of its factors.

\section{Methods}

Process of developing the questionnaire

We first created 47 items to evaluate the PCA and AL.
Two authors who had been teaching AL to workers for more than five years created the initial items together. Factors that were considered at that time were Rogers' three elements, technical aspects of doing $\mathrm{AL}$, and whether or not AL is actually utilized. Each item was made so that it reflected one of these factors. When writing sentences of the items, we took into account our experience in teaching workers in our AL training program, and comments and responses given by participants. However, we did not include expressions that were closely related to the training program itself, considering that the questionnaire might be used in situations independent of the program.

Every item had four response alternatives, the basic pattern of which was "agree", "rather agree", "rather disagree", and "disagree". Respondents were instructed to choose one answer out of the four alternatives based on their ordinary style of listening in the workplace during the past one month.

Second, 83 students (64 male and 19 female) who participated in the postgraduate basic training course of occupational medicine at our university were asked to fill in the questionnaire. Their average age was $27.7 \mathrm{yr}$. We then analyzed the data to determine whether or not items had markedly disproportionate responses to the alternatives. When the number of responses to a certain alternative of an item was null or very small, we modified the expression of that item and/or its alternatives. Several modifications were made to the first questionnaire in this way.

Finally, the modified questionnaire was used to obtain data with which to conduct a factor analysis of the items. All items are listed in the Appendix. We asked 536 workers at two manufacturing companies to fill in the questionnaire and received responses from all workers. Their average age was $34.6 \mathrm{yr}$; 426 were male, 95 were female, and 15 did not specify gender.

\section{Process of factor analysis}

Response alternatives of "agree", "rather agree", "rather disagree", and "disagree" were scored as 3, 2, 1, and 0 , respectively. A factor analysis was then conducted by using PROC FACTOR of SAS ${ }^{12 !}$. As a first step, Kaiser's Measure of Sampling Adequacy ${ }^{13 ;}$ was calculated to judge whether or not each item could be used appropriately in the analysis. This process selected 40 items, and only the data from the 481 respondents who had responded to all of these items were used in the following analyses.

As the next step, responses to the items were subjected to an exploratory factor analysis using squared multiple correlations as prior communality estimates. The principal factor method was used to extract the factors. In this step, the analysis was conducted without any restriction as to the number of factors, and then a scree 
test ${ }^{1+3}$ was used to obtain the number of meaningful factors.

As a final step, the principal factor method was repeated under the condition in which the number suggested by the scree test was specified as the maximum number of factors to be extracted. This was followed by a varimax rotation. In interpreting the rotated factor pattern of the final analysis, an item was said to load on a given factor if the factor loading was 0.40 or greater for that factor and was less than 0.40 for the others.

For factor scores, we could use two scoring approaches: an estimated factor score and a factor-based score ${ }^{|5|}$. The former is a linear composite of the optimally weighted items under analysis, and the latter is a linear composite of the items that demonstrated meaningful loadings for the factor in question. Because we used factor-based scores, each score was calculated only from the questionnaire items that had high loadings on the factor. For a given respondent, his/her score was the sum of his/ her responses to such items. Scores calculated in this way were designated as subscale scores in this study. To interpret scores in terms of $\mathrm{AL}$, when respondents with a high PCA were expected to score low on the original score of a factor, its subscale score was calculated by reversing the scores of all its items. Consequently, respondents with high PCA could score high on all factors.

\section{Assessment of reliability and validity}

After the final factor structure was determined, the reliability and validity of each factor were examined. The reliability of the factors was tested in two ways: internal consistency reliability using Cronbach's alpha ${ }^{16 !}$ and testretest reliability ${ }^{17)}$. For the test-retest reliability calculation, 61 employees (all males with an average age of $51.0 \mathrm{yr}$ ) at a local government office who had no knowledge of the PCA or AL responded to the questionnaire twice at an interval of two weeks.

Validity was examined by comparing the subscale scores of the 536 workers with those of doctors and psychologists specializing in psychosomatic medicine who were working for hospitals or private clinics. Therapists in this field were expected to have an attitude of listening well to patients or clients as a basis of psychotherapy. We asked seven experienced therapists, who were working or had worked for the department of psychosomatic medicine at Kyushu University Hospital and were currently in charge of their respective establishments, to choose doctors and psychologists whom they thought had sufficient clinical experience. The therapists were then asked to fill in the questionnaire. We obtained data from 39 therapists (28 male and 11 female) whose average age was $41.2 \mathrm{yr}$. The subscale scores of the therapists were compared with those of the workers' in a two-sample $t$ test.

\section{Results}

Factor structure of the questionnaire

The initial principal factor analysis on the 40 scale items indicated that the first six eigenvalues and their explained percentages of variance were $6.01(41.1 \%)$, 4.11 (28.1\%), 1.76 (12.0\%), 0.88 (6.0\%), 0.81 (5.6\%), and $0.73(5.0 \%)$. Changes in the eigenvalues and percentages of explained variance were substantive for the first through the fourth factors, but were very small from the fourth through all subsequent factors. The scree test suggested three meaningful factors, so only these factors were retained for rotation. The cumulative contribution of the three factors was $81.2 \%$. A varimax rotation was conducted for these three common factors. Table I shows the items and the factor structure after the rotation. Factor loadings with an absolute value greater than 0.40 are regarded as substantial and are typed in boldface.

The 13 items that had high loadings on the first factor included "I tend to persist in my opinion", "I hurry him/ her into talking faster", "I tend to talk in a directive and persuasive way", "I get irritated from not understanding his/her feelings", "I see him/her from a critical viewpoint", and so forth. Respondents who answered positively to these items were considered to have an attitude completely opposite to the PCA. This factor was labeled "Listening Attitude"; however, in calculating subscale scores, responses to the "Listening Attitude" scale items were reversed, so that respondents with higher PCA would have the higher values.

The second factor, "Listening Skill", was defined by 11 items such as "I pay attention to his/her unexpressed feelings". "I tend to listen to others seriously", "I summarize in my mind what he/she has said", and "I give him/her a brief summary of what he/she has said". Respondents who gave positive answers to these were considered to have listening skills that are consistent with AL.

The seven items that had high loadings on the third factor were "I'm willing to say something to others usually", "People feel easy to talk to me", "I talk with others personally", and so forth. Respondents who replied positively to these items were expected to have many opportunities to talk with their colleagues and subordinates. This factor was labeled "Conversation Opportunity".

The three subscales were constructed using these factors.

\section{Reliability and validity}

The Cronbach's alphas and the test-retest reliabilities of the subscales are shown in Table 2. All three subscales showed high reliability in both methods. In particular, the reliability of the "Listening Attitude" was greater than 0.80 in both calculations, which was the highest among 
Table 1. Questionnaire items and corresponding factor loadings after varimax rotation

\begin{tabular}{|c|c|c|c|c|}
\hline No & Items & Factor 1 & Factor 2 & Factor 3 \\
\hline 33 & I tend to persist in my opinion & 0.65 & -0.01 & 0.15 \\
\hline 28 & I hurry him/her into talking faster & 0.64 & 0.01 & 0.06 \\
\hline 32 & I tend to talk in a directive and persuasive way & 0.60 & 0.12 & 0.12 \\
\hline 34 & I get irritated from not understanding his/her feelings & 0.55 & -0.03 & -0.14 \\
\hline 18 & I see him/her from a critical viewpoint & 0.55 & -0.13 & -0.04 \\
\hline 31 & I tend to deny his/her opinion & 0.54 & -0.10 & -0.08 \\
\hline 27 & I talk about what I want to say, even if I interrupt him/her & 0.54 & -0.09 & 0.28 \\
\hline 12 & I begin arguing with him/her before I know it & 0.52 & 0.15 & 0.07 \\
\hline 35 & I talk offensively when I'm in a bad mood & 0.49 & -0.17 & -0.05 \\
\hline 9 & I begin to talk before he/she finishes talking & 0.49 & -0.14 & 0.22 \\
\hline 13 & I tend to talk, sticking to his/her trivial words & 0.47 & 0.03 & -0.13 \\
\hline 8 & I talk longer than him/her in spite of my intention & 0.41 & -0.04 & 0.37 \\
\hline 45 & Listening to others arouses resistance in me & 0.39 & -0.13 & -0.39 \\
\hline 7 & I understand a person as the stereotype of such and such & 0.36 & -0.07 & -0.03 \\
\hline 43 & I regret that I should not have listened to him/her & 0.34 & 0.01 & -0.22 \\
\hline 15 & When I can't follow, I pretend to understand him/her & 0.30 & -0.28 & -0.18 \\
\hline 44 & I don't get tired from listening to others & -0.33 & 0.18 & 0.22 \\
\hline 36 & When I began to talk at the same time, I let him/her talk & -0.34 & 0.20 & -0.30 \\
\hline 30 & I can listen to him/her, even if he/she has a different opinion & -0.45 & 0.36 & 0.11 \\
\hline 39 & I pay attention to his/her unexpressed feelings & -0.10 & 0.62 & 0.08 \\
\hline 26 & I tend to listen to others seriously & -0.26 & 0.52 & 0.08 \\
\hline 19 & I summarize in my mind what he/she has said & 0.00 & 0.52 & 0.11 \\
\hline 20 & I give him/her a brief summary of what he/she has said & 0.23 & 0.51 & 0.30 \\
\hline 16 & I listen to him/her, putting myself in his/her shoes & -0.09 & 0.50 & 0.18 \\
\hline 38 & When he/she is hesitating, I give him/her a chance & 0.05 & 0.46 & 0.12 \\
\hline 11 & I listen to him/her calmly, while he/she is speaking & -0.27 & 0.44 & 0.07 \\
\hline 47 & I'm pleased that I have given him/her some advice & -0.10 & 0.43 & 0.32 \\
\hline 40 & I pay attention to the changes of his/her feelings & 0.15 & 0.42 & 0.06 \\
\hline 42 & I'm aware of my own feelings & 0.01 & 0.40 & 0.00 \\
\hline 29 & I keep listening to him/her. even if I'm not interested & -0.32 & 0.40 & 0.12 \\
\hline 41 & I'm careful not to interrupt his/her talk & -0.35 & 0.36 & -0.07 \\
\hline 37 & When he/she is hesitating, I wait for him/her to begin talking & -0.30 & 0.32 & -0.27 \\
\hline 14 & I listen to him/her absent-mindedly & 0.32 & -0.41 & -0.07 \\
\hline 5 & I'm willing to say something to others usually & -0.02 & 0.21 & 0.66 \\
\hline 3 & People feel easy to talk to me & 0.08 & 0.11 & 0.58 \\
\hline 1 & I talk with others personally & 0.07 & 0.22 & 0.51 \\
\hline 2 & I'm asked my advice by others & 0.15 & 0.28 & 0.49 \\
\hline 17 & I express my feelings straightforwardly & -0.01 & 0.18 & 0.41 \\
\hline 46 & I can listen to his/her worries, but I can't confide mine & 0.08 & 0.02 & -0.43 \\
\hline \multirow[t]{2}{*}{4} & I don't talk with someone else unless I have something to say & 0.16 & -0.06 & -0.48 \\
\hline & Variance explained by each factor & 5.15 & 3.56 & 2.96 \\
\hline
\end{tabular}

Rotated factor loadings that were greater than 0.40 before rounding up are in boldface.

the three. As shown in Table 3, all the subscale scores differed significantly between the workers and the therapists, with the therapists scoring higher than the workers on each subscale. The differences were especially remarkable on the "Listening Attitude" and "Listening Skill" subscales.

\section{Discussion}

The ALAS developed in this study measures the attitudes of people with respect to a "person-centered attitude" and "active listening". All the items allocated to "Listening Attitude" were those that were initially 
Table 2. Reliability of the three subscales

\begin{tabular}{llcc}
\hline \multicolumn{1}{c}{ Subscale } & Cronbach's alphai & Test-retest reliability' \\
\hline 1 & Listening attitude & 0.84 & 0.83 \\
2 & Listening skill & 0.78 & 0.83 \\
3 & Conversation opportunity & 0.74 & 0.79 \\
\hline
\end{tabular}

aCronbach's alphas were calculated from data from 536 workers. 'The test-retest reliability, with a two-week interval, was examined using data from 61 local government employees.

Table 3. Comparison of subscale scores of workers with those of therapists

\begin{tabular}{|c|c|c|c|c|c|c|}
\hline \multirow{2}{*}{\multicolumn{2}{|c|}{ Subscale }} & \multicolumn{2}{|c|}{ Workers } & \multicolumn{2}{|c|}{ Therapists } & \multirow{2}{*}{$\mathrm{p}^{\mathrm{a}}$} \\
\hline & & Mean & (SD) & Mean & (SD) & \\
\hline 1 & Listening attitude & 24.4 & $(5.4)$ & 29.4 & $(4.9)$ & 0.0001 \\
\hline 2 & Listening skill & 20.3 & $(4.0)$ & 25.0 & (3.3) & 0.0001 \\
\hline 3 & Conversation opportunity & 11.4 & $(3.5)$ & 13.2 & (4.1) & 0.0028 \\
\hline
\end{tabular}

a Differences were analyzed using a two-sample $t$ test. The test was two-tailed.

constructed to relate either to "empathic understanding" or to "unconditional positive regard". Although it did not include items related to "congruence", this subscale was considered to be very similar to what we had expected to find. Regarding the basic attitudes of experts in psychotherapy and psychosomatic medicine, both acceptance and empathy have been emphasized in Japan. This attitude is identical with a combination of "unconditional positive regard" and "empathic understanding", and agrees with the first subscale, which implies that this subscale seems to be acceptable.

Regarding "Listening Skill", eight out of eleven items were selected from those describing technical aspects of $\mathrm{AL}$, whereas the rest of them were related to "empathic understanding" (Item 16), "congruence" (Item 42), or the utilization of AL (Item 47). In our experience, people tend to become aware of the importance of "congruence" when they are very careful about how to listen to others, i.e. the technical aspects of AL. In addition, the item related to "empathic understanding" could also be interpreted as reflecting the technical aspects. All the items on this subscale were therefore considered to be practically comparable with the instructions we give to participants in our AL training programs, which seemed to indicate that the second subscale was consistent with "Listening Skill". Accordingly, at least the first and second subscales could be regarded as having good face validity.

Five out of seven items included in "Conversation Opportunity" belonged to the utilization of AL. One of the remaining items was concerned with "unconditional positive regard" (Item 3 ) and the other with the technical aspects (Item 17). Because the former item could be interpreted to show the attitude of people who frequently converse with others, there seemed to be no remarkable inconsistency in the third subscale. We considered that people who understood the importance of PCA and AL would tend to have many opportunities to use their $\mathrm{AL}$ skills. Hence, the "Conversation Opportunity" subscale seemed to have an indirect relationship with the PCA and AL.

All subscales had Cronbach's alphas of 0.74 or greater. This indicated that each subscale, based on factors extracted, has a satisfactory level of internal consistency. The test-retest reliabilities, with a two-week interval, were also high, being close to or greater than 0.80 for all three subscales. The three subscales are therefore highly reliable measures.

The therapists received significantly higher scores than the lay workers on all the subscales. In particular, the differences were marked for the "Listening Attitude" and "Listening Skill" subscales. Because the therapists had been trained in psychotherapy, it was expected that they would have better listening abilities; therefore, the differences in subscale scores between the two groups are reasonable, suggesting that these subscales have construct validity. Although the scores for "Conversation Opportunity" also differed significantly between the two groups, the level of difference was smaller than those for the other subscales. This might indicate that the construct validity of this subscale is a little lower than that of the others.

When comparing the responses of therapists with those of workers, we did not take into account the influence of 
age and gender, but these might have affected the comparisons. We therefore confirmed the results by using different statistical strategies to compare the two groups. These included comparing the differences after adjusting for age and gender, comparing them separately in each gender, and so forth. None of these provided different results.

In our training program, hardly any participants knew Rogers' three elements before taking part in the program. Sometimes, there were a few workers whose natural listening style was consistent with $\mathrm{AL}$, but it was rare even for those participants to be conscious of the elements. In view of these experiences, it is comprehensible that the three elements were not clearly separated. Nevertheless, we believe that the fact that the analysis revealed such factors as "Listening Attitude" and "Listening Skill" means that Rogers" idea is of universal significance.

However, this does not confirm whether the present factor structure is definite or not, because we do not have any evidence for it in a sample of experts. If we attempt to resolve the question whether or not Rogers' three elements are clearly differentiated, we should examine the attitudes of psychotherapists. This will require obtaining data from more than 200 therapists for a questionnaire of 40 items to comply with the recommendation that the minimum number of subjects in the sample be either greater than 100 or five times the number of variables being analyzed ${ }^{18}$. We need to study this further in order to answer the question, although it may be very difficult to conduct such a study. For the time being, we propose that, because our primary purpose was to measure the individual differences in interpersonal attitude relating to $\mathrm{AL}$ in non-clinical settings, it is justifiable to use lay persons, such as workers, as a target group to standardize the ALAS.

In terms of application, we expect that the ALAS will enable us to evaluate the listening attitudes of workers in a very conventional fashion. It will provide useful information about the attitudes of participants in our training programs, although it does not ask any questions that are directly connected to the program. We also believe that it will be useful in many other areas in which human relationships play an important role, because, according to Rogers, PCA is said to be frequently observed among people who have constructive relationships.

Several issues remain to be considered for its practical utilization. One of its most important applications must be to measure changes in intra-personal listening attitudes after AL training. Even if the questionnaire were confirmed as a useful instrument to clarify the interpersonal differences in attitudes between therapists and workers, we must study further whether or not it is sensitive enough to explain the changes within the same individuals when it is used repeatedly. Whether or not it applies to studying attitudes among therapists should also be investigated.

Another issue to be addressed is that the ALAS could not eliminate the so-called social desirability bias. Because of experiences we typically have in our AL training sessions, we believe that it would not affect the responses of workers very much. Listening to others with the attitudes of AL seems to be very different from workers' usual attitudes in the workplace, one of which is typically represented by 'giving advice to others'. As a consequence, participants in our training program seem to have difficulty in understanding what attitudes are consistent with AL, even after the basic concept of AL is explained to them. This means that lay workers may not be able to recognize which alternative is socially desirable, even if they attempt to choose a desirable one. Although the influence of the social desirability bias on the responses to the ALAS may be more conspicuous after learning $A L$, it does not seem to be a serious problem, at least when the instrument is used with a group of lay people for the first time.

However, the social desirability bias might affect response patterns when the questionnaire is used to measure the attitudes of therapists because they can recognize which alternative is desirable. This issue must be studied in detail, along with the applicability of the ALAS to research into the attitudes of therapists. In addition, the characteristics of respondents, such as age and gender, that may influence the subscale scores must also be clarified. We will investigate some of these issues in our next study.

In conclusion, we do not insist that validity of the ALAS has been proved beyond doubt, nor that the influence of social desirability is small. Because we have not yet obtained definite answers to these questions, we need to clarify features of the ALAS in various ways. For instance, we must study its relationships with other psychometric tests and scales as well as its potential applications. Although our new questionnaire still has many questions to be answered before it becomes widely used, we believe that it provides us with a promising new instrument that can measure the attitudes of $\mathrm{AL}$ among general workers.

Acknowledgments: The authors are deeply grateful to Dr Noboru Iwata for his helpful comments on the initial manuscript. This study was partly supported by a grant from the Japan Ministry of Labour.

\section{References}

1) Shimizu Y, Makino S, Takata T. Employee status during the past decade (1982-1992) based on a nation-wide survey conducted by the Ministry of Labour in Japan. Industrial Health 1997; 37: 441-450. 
2) Hurrel JJ, McLaney MA. Exposure to job stress: a new psychometric instrument. Scan J Work Environ Health 1988; 14 (suppl 1): 27-28.

3) Ikemi A, Kubota S, Noda E, et al. Person-centered approach in occupational mental health; theory, research and practice. Jpn J Ind Health 1992; 34: 1829.

4) Rogers C. The necessary and sufficient conditions of therapeutic personality change. J Consulting Psychology 1957; $21: 95-103$.

5) Rogers C. Client-centered therapy. Boston: HoughtonMifflin, 1969.

6) Rogers C. Active listening. Chicago: The Industrial Relations Center of the University of Chicago, 1955.

7) Japan Industrial Safety and Health Association (JISHA). Textbook for training psychological consultants: health promotion for mind and body (THP). Tokyo: Japan Industrial Safety and Health Association, 1990.

8) Kubota S, Mishima N, Ikemi A, et al. A research in the effects of active listening on corporate mental health training. J Occup Health 1997; 39: 274-279.

9) Gendlin ET. A theory of personality change. In: Worchel \& Byrne eds. Personality change. John Wiley \& Sons, 1964

10) Klein M, Mathieu P, Gendlin E, et al. The experiencing scalc: a research and training manual, Vol. 1 .

\section{Appendix}

1. I talk with others personally.

2. I'm asked my advice by other people.

3. I' $m$ the kind of person whom people feel easy to talk to.

4. I don't talk with someone else unless I have something I have to talk about.

5. I'm willing to say something to others usually.

6. I should listen to others more seriously.

7. I understand a person as the stereotype of such and such.

8. I' $m$ actually talking longer than the other person in spite of my intention to listen to him/her.

9. I begin to talk before the other person finishes talking.

10. I don't think I have a smooth conversation, when the other person becomes silent during talking.

11. I listen to the other person calmly, while he/she is speaking.

12. I begin arguing with the other person before I know it, while I'm listening to him/her.

13. While listening, I tend to talk to the other person, sticking to his/her trivial words.

14. I listen to others absent-mindedly.
Wisconsin: Wisconsin Psychiatric Institute, 1970.

11) Ikemi A, Kira Y, Murayama S, et al. Rating the process of experiencing: the development of a Japanese version of the experiencing scale. Japanese Journal of Humanistic Psychology 1986; 4: 50-61.

12) SAS/STAT software: user's guide. Tokyo: SAS Institute Japan Inc, 1993: 463-508.

13) Cerny BA, Kaiser HF. A study of a measure of sampling adequacy for factor-analytic correlation matrices. Multivariate Behavioral Research 1977; 12: 43-47.

14) Cattell RB. The scree test for the number of factors. Multivariate Behavioral Research 1966; 1: 245-276.

15) Hatcher L. Chapter 1 , Principal component analysis Step 5: creating factor scores or factor-based scores. In: A step-by-step approach to using the SAS system for factor analysis and structural equation modeling. North Carolina: SAS Institute Inc., 1994; 31-39.

16) Cronbach LJ. Coefficient alpha and the internal structure of tests. Psychometrica 1951; 16: 297-334.

17) Guttman L. A basis for analyzing test-retest reliability. Psychometrica 1945; 10: 255-282.

18) Hatcher L. Chapter 1, Principal component analysis example: analysis of the Prosocial Orientation Inventory. In: A step-by-step approach to using the SAS system for factor analysis and structural equation modeling. North Carolina: SAS Institute Inc., 1994; $10-13$.
15. When I can't follow what the other person is talking about, I pretend to understand it.

16. I listen to the other person, putting myself in his/her shoes.

17. I express my feelings straightforwardly.

18. I inadvertently see the other person from a critical viewpoint.

19. I listen to the other person, summarizing in my mind what he/she has said.

20. I sometimes give the other person a brief summary of what he/she has said.

21. I never turn down another person's request to give him/her some advice.

22. I become emotional in spite of myself, while I'm talking.

23. I can mutually understand anybody.

24. I can take an interest in anybody.

25. I tell the other person whatever things I feel.

26. I tend to listen to others seriously.

27. When I want to say something, I talk about it, even if I interrupt the other person.

28. I tend to hurry the other person into talking faster.

29. I can keep listening to the other person, even if I'm 
not interested in his/her talk.

30. I can listen to the other person, even if he/she has a different opinion from mine.

31. I tend to deny the other person's opinion, when it's different from mine.

32. I tend to talk in a directive and persuasive way, while talking with others.

33. I tend to persist in my opinion, while talking with others.

34. While listening, I get irritated from not understanding the other person's feelings.

35. I talk offensively, when I'm in a bad mood.

36. When I began to talk at the same time as the other person did, I let him/her talk.

37. When the other person is hesitating, I wait for him/ her to begin talking.

38. When the other person is hesitating, I give him/her a chance by saying "For example, is it like this?"
39. I listen to the other person, paying attention to his/ her unexpressed feelings.

40. I listen to the other person, paying more attention to the changes of his/her feelings than to the contents of his/her talk.

41. While listening, I'm careful not to interrupt the other person's talk.

42. I'm aware of my own feelings, while I'm listening to others.

43. After the conversation, I regret that I should not have listened to the other person.

44. I don't get tired from listening to others.

45. Listening to others arouses resistance in me.

46. I can listen to other persons' worries, but I can't confide mine.

47. I'm pleased that I have given some advice to the other person. 